\title{
Acute clinical adverse radiation effects after Gamma Knife surgery for vestibular schwannomas
}

\author{
Constantin Tuleasca, MD,1-3 Mercy George, MD, ${ }^{3,4}$ Mohamed Faouzi, PhD, ${ }^{3,5}$ \\ Luis Schiappacasse, MD, ${ }^{3,6}$ Henri-Arthur Leroy, MD, ${ }^{1,7}$ Michele Zeverino, MSc, ${ }^{8}$ \\ Roy Thomas Daniel, MCh, ${ }^{1,3}$ Raphael Maire, MD, ${ }^{3,4}$ and Marc Levivier, MD, PhD, IFAANS ${ }^{1,3}$
}

${ }^{1}$ Department of Clinical Neurosciences, Neurosurgery Service and Gamma Knife Center, ${ }^{4}$ Department of ENT Surgery, ${ }^{5}$ nstitute of Social and Preventive Medicine, and ${ }^{6}$ Radiation Oncology Service, Lausanne University Hospital (CHUV), Lausanne; ${ }^{2}$ Signal Processing Laboratory (LTS 5), Ecole Polytechnique Fédérale de Lausanne (EPFL); ${ }^{3}$ Faculty of Biology and Medicine, University of Lausanne, Switzerland; ${ }^{7}$ Division of Neurosurgery, Centre Hospitalier Régional Universitaire de Lille, France; and ${ }^{8}$ nnstitute of Radiation Physics, Lausanne, Switzerland

OBJECTIVE Vestibular schwannomas (VSs) represent a common indication of Gamma Knife surgery (GKS). While most studies focus on the long-term morbidity and adverse radiation effects (AREs), none describe the acute clinical AREs that might appear on a short-term basis. These types of events are investigated, and their incidence, type, and outcomes are reported in the present paper.

METHODS The included patients were treated between July 2010 and March 2016, underwent at least 6 months of follow-up, and presented with a disabling symptom during the first 6 months after GKS that affected their quality of life. The timing of appearance, as well as the type of main symptom and outcome, were noted. The prescribed dose was 12 Gy at the margin.

RESULTS Thirty-five (22\%) of 159 patients who fulfilled the inclusion criteria had acute clinical AREs. The mean followup period was 30 months (range 6-49.2 months). The mean time of appearance was 37.9 days (median 31 days; range $3-110$ days). In patients with de novo symptoms, the more frequent symptoms were vertigo $(n=4 ; 11.4 \%)$ and gait disturbance $(n=3 ; 8.6 \%)$. The exacerbation of a preexisting symptom was more frequently related to hearing loss $(n=10$; $28.6 \%)$, followed by gait disturbance $(n=7 ; 20 \%)$ and vertigo $(n=3,8.6 \%)$. In the univariate logistic regression analysis, the following factors were statistically significant: age ( $p=0.002$; odds ratio [OR] 0.96), hearing at baseline by GardnerRobertson (GR) class ( $p=0.006$; OR 0.21), pure tone average at baseline ( $p=0.006 ;$ OR 0.97), and Koos grade at baseline (with Koos Grade I used as a reference) (for Koos Grade II, OR 0.17 and p = 0.002; for Koos Grade III, OR 0.42 and $p=0.05$ ). The following were not statistically significant but showed a tendency toward significance: the number of isocenters ( $p=0.06$; OR 0.94) and the maximal dose received by the cochlea ( $p=0.07 ;$ OR 0.74$)$. Fractional polynomial regression analysis showed a nonlinear relationship between the outcome and the radiation dose rate (minimum reached at a cutoff of $2.5 \mathrm{~Gy} /$ minute) and the maximal vestibular dose (maximum reached at a cutoff of $8 \mathrm{~Gy}$ ), but the small sample size precludes a detailed analysis of the former. The clinical acute AREs disappeared in 32 (91.4\%) patients during the first 6 months after appearance. Permanent and somewhat disabling morbidity was found in 3 cases $(1.9 \%$ from the whole series): 1 each with complete hearing loss (GR Class I before and V after), hemifacial spasm (persistent but alleviated), and dysgeusia.

CONCLUSIONS Acute effects after radiosurgery for VS are not rare. They concern predominantly de novo vertigo and gait disturbance and the exacerbation of preexistent hearing loss. In de novo vestibular symptoms, a vestibular dose of more than $8 \mathrm{~Gy}$ is thought to play a role. In most cases, none of these effects are permanent, and they will ultimately improve or disappear with steroid therapy. Permanent AREs remain very rare.

http://thejns.org/doi/abs/10.3171/2016.7.GKS161496

KEY WORDS radiosurgery; Gamma Knife surgery; vestibular schwannoma; adverse radiation effects; hearing; gait; loss; disturbance; stereotactic radiosurgery

ABBREVIATIONS ARE = adverse radiation effect; $\mathrm{CN}=$ cranial nerve; GKS = Gamma Knife surgery; GR = Gardner-Robertson; $\mathrm{HB}=\mathrm{House}-\mathrm{Brackmann}$; OR = odds ratio; PIV = prescription isodose volume; PTA = pure tone average; $R S$ = radiosurgery; TV = target volume; VS = vestibular schwannoma.

SUBMITTED June 9, 2016. ACCEPTED July 7, 2016.

INCLUDE WHEN CITING DOI: 10.3171/2016.7.GKS161496. 
$\mathrm{V}$ ESTIBULAR schwannomas (VSs) represent a common indication of Gamma Knife surgery (GKS). While most studies focus on long-term morbidity and adverse radiation effects (AREs), none describe the acute clinical AREs that might appear on a short-term basis.

The primary aim of radiosurgery (RS) treatment for VSs is tumor control, which is attained at very high rates. Mainly, for GKS series, the tumor control rates achieved at 5 years are between $89 \%$ and $98 \% .^{1,2,5,19}$ Overall facial nerve preservation rates range between $84 \%$ and $100 \%, 3,5,11,13,16,18$, 19,21 and trigeminal nerve preservation rates range between $74 \%$ and $99 \% .^{3,5,11,13,16,18,19,21}$ Other cranial nerve $(\mathrm{CN})$ neuropathies are extremely rare, which also applies to hydrocephalus, tinnitus, and vertigo. ${ }^{20}$ In the context of a high tumor control rate with minimal morbidity, hearing preservation has become a new challenge during the last decade. Studies focusing on the doses received by the cochlea and their major impact on long-term hearing preservation have opened a new avenue for functional preservation. ${ }^{19}$

The aim of the present study is to evaluate the incidence, type, and outcome of clinical acute AREs that present during the first 6 months after RS, which is defined as a new or previously present symptom (but increased in intensity and/or frequency) and considered by the patient as disabling and affecting their quality of life. The main hypothesis was that besides the dose to the cochlea-which has been demonstrated to play a role in hearing preservation $^{15}$ - other factors might also play a role, including the dose received by the vestibule, the former mainly with regard to vestibular symptoms (increased in and/or developed de novo) after GKS.

\section{Methods \\ Type of Study}

The study was designed as an open, retrospective, nonrandomized study. A case report form was created and prospectively filled in with the baseline and follow-up data.

\section{Participants}

VS is one of the most frequent pathologies treated in our Gamma Knife Center, accounting for almost 30\% of the patients. All patients with a Koos Grade I to III VS and at least 6 months of follow-up who were treated with GKS between July 2010 and March 2016 were included in the study. Of a total of 159 patients (patients with $<6$ months of follow-up, recurrent or residual tumor from previous surgery, neurofibromatosis Type 2, and postradiosurgery and postradiotherapy failure were excluded), 35 (22\%) patients had an acute clinical ARE.

\section{Baseline and Follow-Up Monitoring}

The baseline assessment included recording clinical demographic data and a full clinical examination, including examination of the CNs. Paraclinical examination included vocal and tonal audiometry, caloric testing, and brain MRI. After GKS, patients were evaluated at 6, 12, 24, 36, and 60 months using otoneurological outpatient tests, brain MRI, and neurosurgery consultation.

Auditory function was analyzed using the Gardner-
Robertson (GR) classification. ${ }^{4}$ Facial function was assessed using the House-Brackmann (HB) scale. ${ }^{8}$

\section{Brief Description of the GKS Technique}

After application of the Leksell model G stereotactic frame (Elekta Instruments AB) under local anesthesia, all patients underwent stereotactic MRI and bone CT for the target and organs at risk definition. During dosimetry planning, special attention was given to the dose received by the cochlea in patients with functional hearing at baseline..$^{15}$

An added value of the present study is the definition of the vestibule as an organ at risk: the vestibule was defined as the structure that is very close anatomically to the cochlea and which might be subjected to a dose increase when limiting the dose to cochlea. The vestibule corresponds to the posterior labyrinth and is constituted by a central part-where the otolith organs, the saccule and utricle, are located-that is close to the cochlea and surrounded by the 3 semicircular canals. The saccule is usually a rather small and difficult to identify structure in nonpathological conditions on CT images compared with the utricle, which is easily identifiable and in a reproducible manner. Additionally, the utricle has a consistent volume. Furthermore, in our experience, while protecting the cochlea, the gradient of dose is most frequently deviated towards the utricle. So, we decided to measure the dose delivered to the utricle (see in Fig. 9), which is anatomically located in close proximity to the modiolus.

The MRI sequences used were adapted to the VS size and included T1- and T2-weighted constructed interference in steady-state (CISS)/fast imaging employing steady-state acquisition (FIESTA) sequences, both with and without contrast enhancement; the latter was used for better visualization of the CNs ${ }^{6,7}$ Bone CT routinely supplemented the neuroimaging investigation to correct any distortion errors that might be encountered on MRI. Additionally, bone CT provided important information with regard to the bony landmarks of the internal auditory canal, as well as the cochlea and vestibule.

Sector blocking was frequently used, especially for the shots located in the internal auditory canal, in order to keep the maximal dose to the cochlea below 5.2 Gy.

\section{Definition of Clinical Acute AREs}

Before discharge following GKS, all patients were informed that they should contact us directly if they presented with a disabling symptom that affected their quality of life. All such symptoms that appeared during the first 6 months after GKS (i.e., before the first usual postGKS visit) were prospectively noted on the case report form. The timing of appearance, as well as the types of symptoms and their nature (de novo and/or preexisting but exacerbated in terms of frequency and/or intensity) were noted. All patients were seen in person for a detailed evaluation at that time. For those patients who presented with acute hearing loss, an otoneurological evaluation was also performed.

If a clinical acute ARE was diagnosed, treatment with prednisone $(1 \mathrm{mg} / \mathrm{kg} / \mathrm{day})$ was initiated. The duration of this pharmacological treatment was at least 7 days. After 
this period, a telephone interview and/or outpatient visit was scheduled, including vocal and tonal audiometry for those with new or worsened hearing loss. Prednisone was tapered and stopped if the symptom disappeared, and the patient was then seen again at the regular 6-month followup. If the symptom persisted after this period, brain MRI was scheduled and the patient was additionally seen in the outpatient clinic. For those patients who presented with acute hearing loss, audiometry was performed at the time of appearance and after the end of prednisone treatment.

\section{Statistical Consideration}

Data analysis was performed using Stata software (Stata Statistical Software, release 14; StataCorp LP). Categorical variables were summarized by their frequencies and percentages, and continuous variables were summarized by their mean and standard deviation. Univariate logistic regression was performed to assess the association between each predictor and the outcome (acute clinical ARE). The strength of the association was measured using the odds ratios (ORs) and their associated $p$ values. The relationship between the outcome and continuous variables was checked using fractional polynomials regression modeling. The linearity assumption was confirmed for age, but not for the radiation dose rate, maximal vestibular dose, pure tone average (PTA) at baseline, number of shots, target volume (TV, ml), maximal dose (Gy), maximal dose received by the cochlea, and maximal dose received by the vestibule. Because of the insufficient sample size, we did not perform any multivariate analysis.

\section{Basic Demographic Data}

The mean patient age was 52 years (median 49 years; range 23-82 years). The mean follow-up period was 30 months (range 6-49.2 months). In the present series, 22 (62.9\%) patients were female and $13(37.1 \%)$ patients were male. The type of symptom at the time of the diagnosis of VS included no symptoms (i.e., incidental finding) in $3(8.2 \%)$ patients, hypoacusis in $22(62.9 \%)$ patients (of whom 7 patients had acute hearing loss at discovery), tinnitus in $3(8.6 \%)$ patients, vertigo in $6(17.1 \%)$ patients, and gait disturbances in $1(2.9 \%)$ patient (Table 1$)$.

Hearing status at baseline was GR Class I in $25(71.4 \%)$ patients, GR Class II in $6(17.1 \%)$ patients, GR Class III in $2(5.7 \%)$ patients, GR Class IV in $0(0 \%)$ patients, and GR Class V in $2(5.7 \%)$ patients. Thus, $78.5 \%$ of these patients had functional hearing (GR Class I or II) before GKS. Tonal audiometry displayed a mean PTA of $45.9 \mathrm{~dB}$ and a median of $24.2 \mathrm{~dB}$ (range 3.75-113.75 dB).

Koos grading was Grade I in $16(45.7 \%)$ patients, Grade II in $5(14.3 \%)$ patients, and Grade III in 14 (40\%) patients. The size of the VS at the time of GKS indication was compared with the previous MRI studies performed during the previous months or years. This displayed a discrete increase in volume in $19(54.3 \%)$ patients, stability over the last 12 months in $15(42.9 \%)$ patients, and a rapid increase over the same period in $1(2.9 \%)$ patient.

The mean TV was $0.81 \mathrm{ml}$ (range 0.02-7.8 ml), and the prescription isodose volume (PIV) was $0.94 \mathrm{ml}$ (range 0.04-8.5 ml). The mean number of shots was 7.5 (range $1-29$ ). In our series, we usually prescribe 12 Gy at the $50 \%$ isodose line, in accordance with the dose deescalation studies already published. ${ }^{10}$ However, especially for some cases of Koos Grade I VS, we needed to prescribe a higher isodose, thereby allowing the previously stated variability. The mean maximal dose was 23.5 Gy (range 15-26.67 Gy). The mean maximal dose received by the cochlea was 3.9 Gy (range 1.7-7.6 Gy). The mean maximal dose at the vestibule was 5.3 Gy (range 1.2-13.2 Gy). The mean dose rate was $2.83 \mathrm{~Gy} /$ minute (range $1.88-3.66 \mathrm{~Gy} /$ minute).

\section{Results}

\section{Time to Appearance of Clinical Acute AREs}

The mean time to appearance was 37.9 days (median 31 days; range 3-110 days). The time to appearance of the clinical acute AREs was 3 days in 4 (11.4\%) patients; 4 days in 2 (5.7\%) patients; 7 days in 3 (8.6\%) patients; 8,12 , 15 , or 16 days in 1 patient each (2.9\% multiplied by 4$) ; 30$ days in $4(11.4 \%)$ patients; 31 days in 5 (14.3\%) patients; $40,44,58,59,61,62,78,82$, and 91 days in 1 patient each (2.9\% multiplied by 9); 92 days in 3 (8.6\%) patients, and 110 days in 1 patient (2.9\%). There was a peak in acute symptomatology during the first week, as well as in the 1st and the 3rd months after GKS (Fig. 1).

\section{Type of Clinical Acute AREs After GKS}

De novo symptoms appeared in $42.9 \%$ of patients, while the exacerbation of preexisting symptoms appeared in $57.1 \%$ of patients (Table 2).

Among the de novo symptoms, the predominant symptoms were vertigo ( $\mathrm{n}=4,11.4 \% ; 2.5 \%$ of the entire series) and gait disturbance $(\mathrm{n}=3,8.6 \% ; 1.9 \%$ of the number of VSs treated during the same period of time), followed by hearing loss and tinnitus (each $\mathrm{n}=2,5.7 \% ; 1.2 \%$ of the number of VSs treated during the same period of time), facial palsy, dysgeusia, and hemifacial spasm (each $\mathrm{n}=1$, $2.8 \% ; 0.6 \%$ of the number of VSs treated during the same period of time). Facial palsy was graded as HB Grade III and appeared 1 week after GKS in a patient who already had contrast enhancement on the facial nerve that was seen retrospectively.

The exacerbation of a preexisting symptom was more frequently related to hearing loss $(n=10,28.6 \% ; 6.3 \%$ of the entire series), followed by gait disturbance $(n=7,20 \%$; $4.4 \%$ of the entire series), vertigo $(n=3,8.6 \% ; 1.9 \%$ of the entire series), and tinnitus ( $\mathrm{n}=1,2.8 \% ; 0.6 \%$ of the number of VSs treated during the same period of time).

\section{Evolution of Clinical Acute AREs During the Follow-Up Period}

Clinical acute AREs resolved in $32(91.4 \%)$ of patients during the first 6 months after appearance. Usually, there was a marked alleviation and/or disappearance of the symptoms during the week of corticosteroid treatment.

Regarding the particular types of signs and symptoms, their evolution was as follows. Audiometry evaluation showed that $1(0.6 \%)$ patient did not improve from acute hearing loss with deterioration from GR Class I to GR Class V, and 5 (3.2\% from the whole series) patients moved from GR Class I to GR Class III during the followup course. In total, $82.8 \%$ of patients kept their functional hearing, with the same GR class as at baseline. Further- 
TABLE 1. Basic demographic data

\begin{tabular}{|c|c|c|}
\hline Variable & Acute Clinical AREs & No Acute Clinical AREs \\
\hline \multicolumn{3}{|l|}{ Sex, n (\%) } \\
\hline Male & $22(62.9)$ & $69(55.7)$ \\
\hline Female & $13(37.1)$ & $55(44.3)$ \\
\hline \multicolumn{3}{|l|}{ Symptom at discovery, $\mathrm{n}(\%)$} \\
\hline Incidental & $3(8.6)$ & $10(8.1)$ \\
\hline Hypoacusia & $22(62.9)$ & $80(64.5)$ \\
\hline Tinnitus & $3(8.6)$ & $11(8.9)$ \\
\hline Vertigo & $6(17.1)$ & $17(13.7)$ \\
\hline Gait disturbances & $1(2.9)$ & $3(2.4)$ \\
\hline \multicolumn{3}{|l|}{ Hearing at baseline, $n(\%)$} \\
\hline GR I & $25(71.4)$ & $49(39.5)$ \\
\hline GR II & $6(17.1)$ & $28(22.6)$ \\
\hline GR III & $2(5.7)$ & $21(16.9)$ \\
\hline GR IV & $0(0)$ & $3(2.4)$ \\
\hline GR V & $2(5.7)$ & $23(18.6)$ \\
\hline \multicolumn{3}{|l|}{ Mean PTA (min-max), dB } \\
\hline Baseline & $32.6(7.5-107.5)$ & $45.9(3.7-113.7)$ \\
\hline 6 mos after GKS & $39.8(2.5-110)$ & $49.3(5-111.2)$ \\
\hline 12 mos after GKS & $43.6(2.5-130)$ & $52.9(6.2-111.2)$ \\
\hline 24 mos after GKS & $38.9(2.5-81.2)$ & $56.9(6.2-120)$ \\
\hline 36 mos after GKS & $38.4(2.5-86.2)$ & $58.8(10-113.7)$ \\
\hline \multicolumn{3}{|l|}{ Koos grade at baseline, $n(\%)$} \\
\hline Grade I & $16(45.7)$ & $25(20.2)$ \\
\hline Grade II & $5(14.3)$ & $45(36.3)$ \\
\hline Grade III & $14(40)$ & $51(41.1)$ \\
\hline \multicolumn{3}{|l|}{ Paraclinical evolution before GKS (\%) } \\
\hline Discrete & $19(54.3)$ & $60(48.4)$ \\
\hline Rapid & $1(2.9)$ & $3(2.4)$ \\
\hline None & $26(42.9)$ & $61(49.2)$ \\
\hline Mean maximal dose \pm SD (min-max), Gy & $23.5 \pm 2.04(15-26.7)$ & $23.4 \pm 1.67(16-24)$ \\
\hline Mean no. of shots \pm SD (min-max), Gy & $7.5 \pm 6.92(1-29)$ & $10.1 \pm 7.24(1-32)$ \\
\hline Mean maximal cochlea dose \pm SD (min-max), Gy & $3.9 \pm 1.27(1.7-7.6)$ & $4.4 \pm 1.45(1.5-10.3)$ \\
\hline Mean maximal vestibular dose \pm SD (min-max), Gy & $5.4 \pm 2.87(1.2-13.2)$ & $5.4 \pm 2.43(1.3-12.4)$ \\
\hline Mean target volume $\pm \mathrm{SD}$ (min-max), ml & $0.81 \pm 1.43(0.02-7.8)$ & $1.18 \pm 1.44(0.01-6.4)$ \\
\hline Mean prescription isodose volume \pm SD (min-max), ml & $0.94 \pm 1.55(0.04-8.5)$ & $1.37 \pm 1.59(0.03-6.8)$ \\
\hline Mean radiation dose rate $\pm \mathrm{SD}$ ( $\min -\max ), \mathrm{Gy} / \mathrm{min}$ & $2.83 \pm 0.56(1.88-3.66)$ & $2.79 \pm 0.51(1.88-3.67)$ \\
\hline
\end{tabular}

more, the evolution of the PTA values at $6,12,24$, and 36 months can be seen in Table 1 . Nonauditory morbidity evolved as follows: facial palsy disappeared at 3 months in a patient with an HB Grade I persisting at 3 years after GKS; the hemifacial spasms have decreased in intensity and frequency, but persist at 2 years after GKS; dysgeusia remained constant in the only patient with this symptom; and gait problems persisted in only 1 patient at 3 years, but these were not symptoms that interfered with quality of life.

\section{Tumor Control at the Last Follow-Up}

At the last follow-up, all tumors (with 1 exception) were either stable or had decreased in size. The 1 excep- tion, which displayed an increase at 6 and 12 months, is a Koos Grade I VS in a patient who had clinically persistent disease and a disabling hemifacial spasm. The patient has now received 3 years of follow-up, and the tumor corresponds to Koos Grade II. Decompressive surgery was initially proposed to the patient for symptomatic reasons with the aim of alleviating the hemifacial spasms, but this was declined by the patient. Because there was MRI stability over a period of 3 years, the decision to wait and scan has now been adopted.

\section{The Need for Further MRI Evaluation}

After 1 week of pharmacological treatment, MRI was performed in $3(8.6 \%)$ patients due to the persistence of 


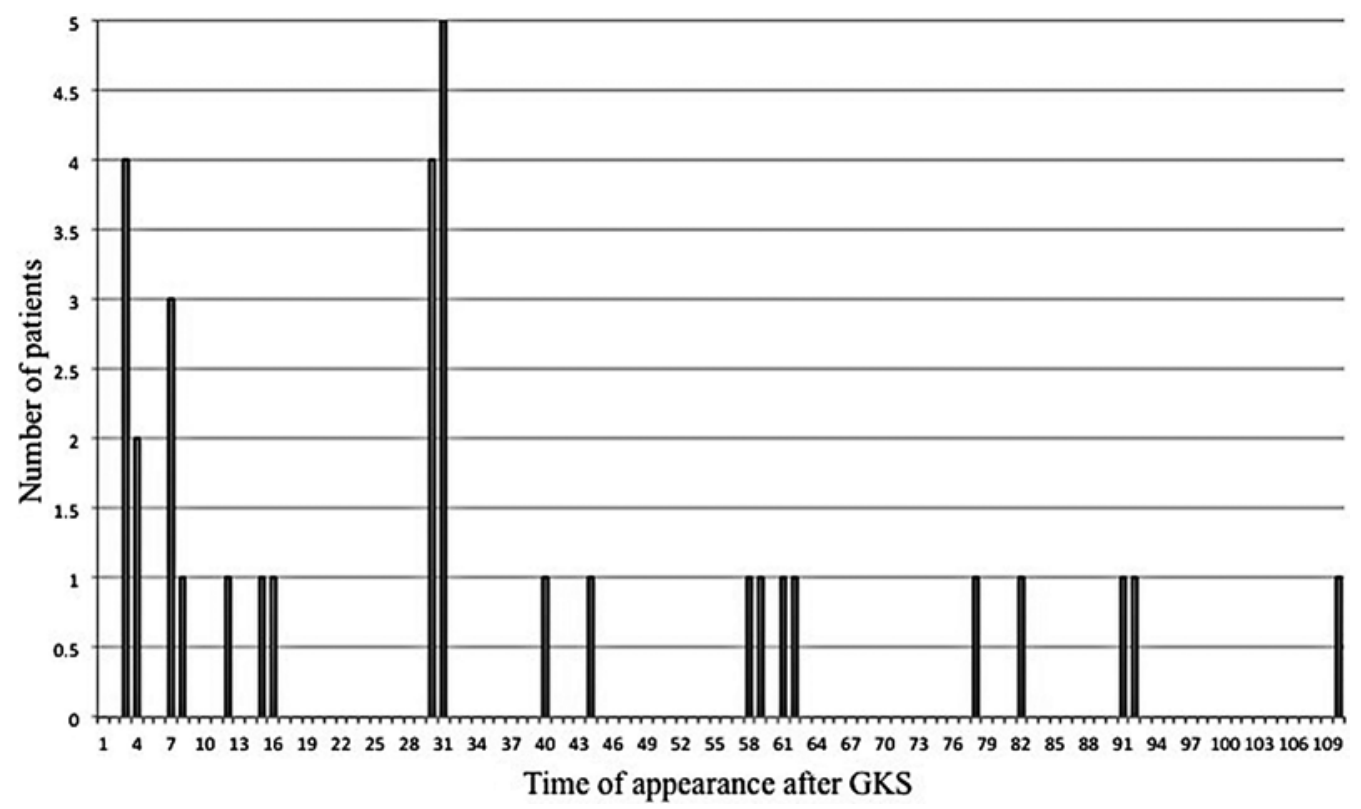

FIG. 1. Time of appearance (in days) of an acute effect after GKS.

symptoms with similar intensity as reported before corticosteroids.

One MRI session was performed at 1 month, and 2 MRI sessions were performed at 3 months. Tumor progression was not found in any case, and the T2-weighted sequence exhibited no edema or other side effect. There was no hydrocephalus.

\section{Predictors of Clinical Acute AREs: Univariate Analysis}

In the univariate logistic regression analysis (Table 3), the following factors were significantly associated with outcome: age ( $\mathrm{p}=0.002$; OR 0.96; Fig. 2); hearing level at baseline (by GR class; $p=0.006$; OR 0.21); PTA at baseline ( $\mathrm{p}=0.006$; OR 0.97; Fig. 3); and Koos grade at base-

TABLE 2. Type of acute clinical ARE after GKS (coded as the dominant symptom)

\begin{tabular}{lc}
\hline \multicolumn{1}{c}{ Variable } & No. of Patients $(\%)$ \\
\hline De novo symptom* & \\
\hline Hearing loss & $2(5.7)$ \\
\hline Tinnitus & $2(5.7)$ \\
\hline Vertigo & $4(11.4)$ \\
\hline Gait disturbance & $3(8.6)$ \\
\hline Facial palsy & $1(2.8)$ \\
\hline Dysgeusia & $1(2.8)$ \\
\hline Facial hemispasm & $1(2.8)$ \\
\hline Aggravation of a preexisting symptom $\dagger$ & \\
\hline Hearing loss & $10(28.6)$ \\
\hline Tinnitus & $1(2.8)$ \\
\hline Vertigo & $3(8.6)$ \\
\hline Gait disturbance & $7(20)$ \\
\hline
\end{tabular}

* Affected $42.9 \%$ of all patients.

$\dagger$ Affected $57.1 \%$ of all patients.
TABLE 3. Results of the univariate logistic regression analysis

\begin{tabular}{|c|c|c|c|}
\hline Variable & OR & $95 \% \mathrm{Cl}$ & $p$ Value \\
\hline Age & 0.96 & $0.928-0.982$ & 0.002 \\
\hline Age by 5 yrs & 0.79 & $0.691-0.917$ & 0.002 \\
\hline Age by 10 yrs & 0.63 & $0.478-0.842$ & 0.002 \\
\hline Sex & 0.74 & $0.342-1.603$ & 0.44 \\
\hline Side & 1.46 & $0.689-3.130$ & 0.31 \\
\hline Symptoms at GKS & & & $>0.05$ \\
\hline GR class at baseline & 0.211 & $0.070-0.636$ & 0.006 \\
\hline \multicolumn{4}{|l|}{ GR class at GKS } \\
\hline I & Reference & & \\
\hline II & 0.42 & $0.153-1.147$ & 0.09 \\
\hline III & 0.18 & $0.040-0.860$ & 0.03 \\
\hline \multicolumn{4}{|l|}{$\mathrm{IV}^{*}$} \\
\hline V & 0.170 & $0.037-0.781$ & 0.02 \\
\hline PTA at baseline & 0.97 & $0.952-0.991$ & 0.006 \\
\hline Koos at baseline & 0.86 & $0.402-1.854$ & 0.7 \\
\hline I & Reference & & \\
\hline II & 0.17 & $0.056-0.530$ & 0.02 \\
\hline III & 0.42 & $0.181-1.015$ & 0.05 \\
\hline Paraclinical evolution before GKS & 0.77 & $0.361-1.668$ & 0.517 \\
\hline Maximal dose, Gy† & 1.04 & $0.831-1.318$ & 0.6 \\
\hline No. of shots $\dagger$ & 0.94 & $0.889-1.003$ & 0.06 \\
\hline Maximal cochlea dose, Gy† & 0.74 & $0.542-1.030$ & 0.07 \\
\hline Cochlea volume $\dagger$ & 0.90 & $0.656-1.250$ & 0.5 \\
\hline Vestibular dose, Gy† & 1.00 & $0.864-1.16$ & 0.9 \\
\hline $\mathrm{TV}, \mathrm{ml}$ & 0.80 & $0.580-1.111$ & 0.18 \\
\hline PIV, ml & 0.81 & $0.602-1.089$ & 0.16 \\
\hline Radiation dose rate, Gy/min & 1.14 & $0.554-2.381$ & 0.7 \\
\hline
\end{tabular}

* Hearing GR Class IV predicts the outcome perfectly because patient with GR Class IV did not have any AREs (see Table 1).

$\dagger$ The relationship to the outcome was forced to be linear (see Figs. 2-8). 


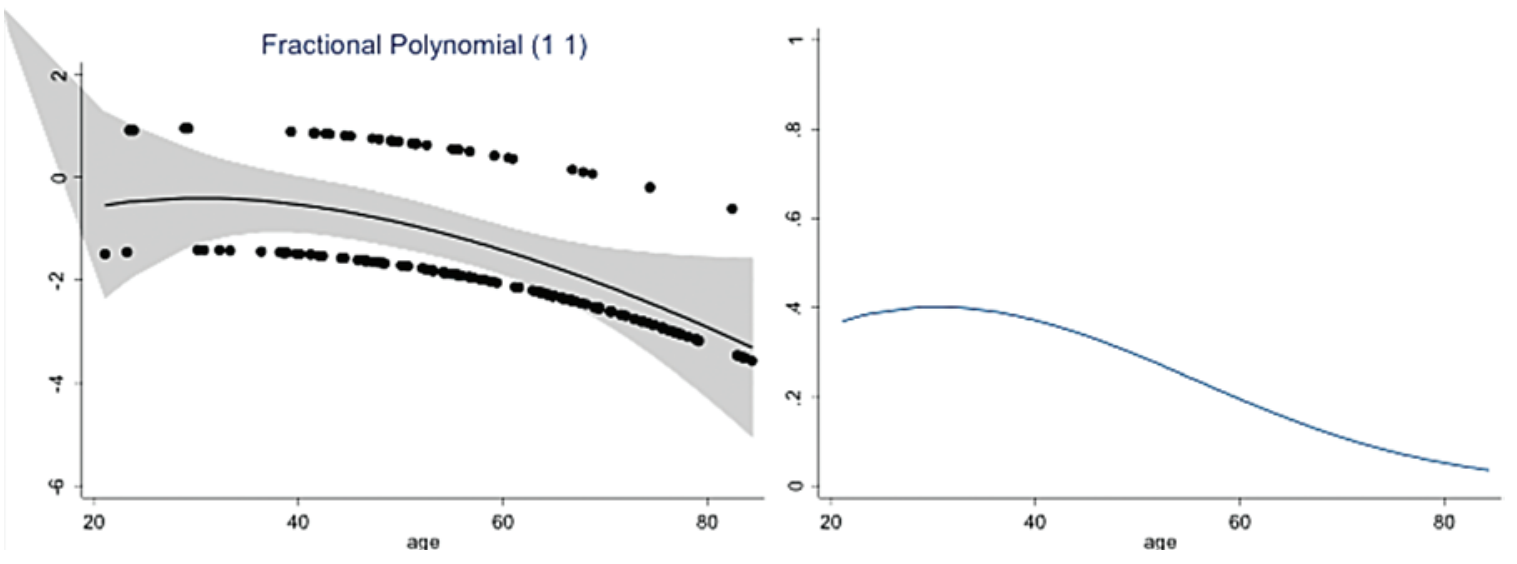

FIG. 2. Probability of presenting an acute effect depending on age at GKS. Left: Probability is shown with confidence intervals. Right: Probability is shown in a simplified manner, ranging from $0 \%$ to $100 \%$.

line $(\mathrm{p}=0.002$; OR 0.17 for Koos Grade II; OR 0.42 for Koos Grade III). Moreover, a tendency toward statistical significance has been shown by the number of isocenters used ( $\mathrm{p}=0.06$; OR 0.94; Fig. 4) and the maximal dose received by the cochlea $(\mathrm{p}=0.07$; OR 0.74$)$. The vestibular dose is shown in Fig. 5.

The following factors were not statistically significant: sex $(\mathrm{p}=0.4$; OR 0.74 for male patients); side $(\mathrm{p}=0.31$; OR 1.46); symptom at baseline ( $p>0.05)$; paraclinical increase before GKS ( $p=0.51$; OR 0.77 for no paraclinical evolution) ( $p=0.96$; OR 1.05 for rapid one); cochlear volume ( $\mathrm{p}=0.54$; OR 0.90); TV ( $=0.18$; OR 0.80; Fig. 6); PIV ( $\mathrm{p}=0.163$; OR 0.81); and dose rate $(\mathrm{p}=0.7$; OR 1.14).

Some factors, including the dose rate and the maximal dose received by the cochlea and vestibule, were further investigated (see below).

\section{Predictors of Clinical Acute AREs: Fractional Polynomial Analysis}

An added value to this study was the statistical analysis using fractional polynomial analysis. The factors used were mainly age, PTA at baseline, maximal dose received by the VS, TV, number of shots, maximal dose received by the cochlea, and the vestibular dose (Table 3).

\section{Maximal Dose Received by the Tumor}

The maximal dose received by the tumor displayed an increase in risk of developing an acute clinical ARE, with a cutoff at $20 \mathrm{~Gy}$. The risk was similar for doses of 17 and $24 \mathrm{~Gy}$, with an exponential increase for more than $24 \mathrm{~Gy}$ (Figs. 6 and 7).

\section{Number of Shots}

The number of shots displayed a cutoff at 5 , with fewer than 5 shots having an increased risk. The risk was the same for 10 or more than 10 shots.

\section{Vestibular Dose}

The vestibular dose displayed a cutoff at $8 \mathrm{~Gy}$. While the risk of developing an acute clinical ARE was low for values below $8 \mathrm{~Gy}$, it increased significantly and continuously for values of more than $8 \mathrm{~Gy}(\mathrm{p}=0.2$ and OR 0.84 for vestibular dose less than $8 \mathrm{~Gy} ; \mathrm{p}=0.6$ and OR 1.14 for vestibular dose more than $8 \mathrm{~Gy}$ ). The maximal dose received by the cochlea displayed a tendency toward statistical significance ( $\mathrm{p}=0.07$; OR 0.74) (Figs. 5, 8, and 9). The significance of the relationship between these 2 factors is further discussed.
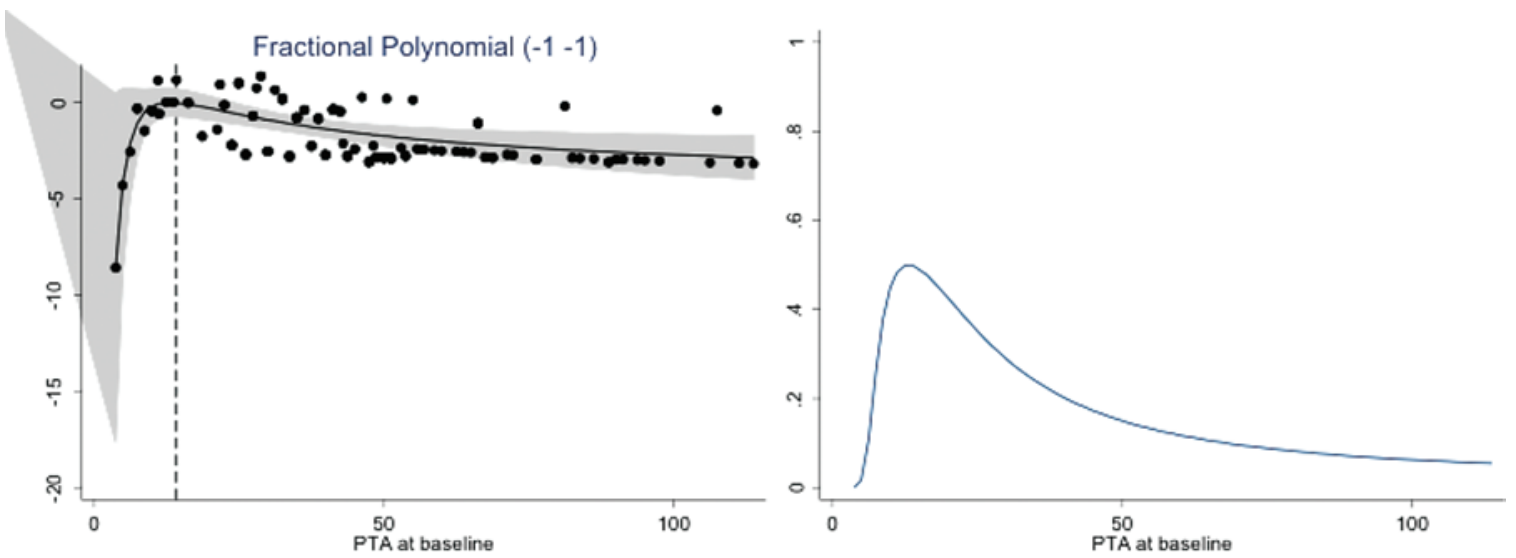

FIG. 3. Probability of presenting an acute effect depending on PTA at GKS. Left: Probability is shown with confidence intervals. Right: Probability is shown in a simplified manner, ranging from $0 \%$ to $100 \%$. 


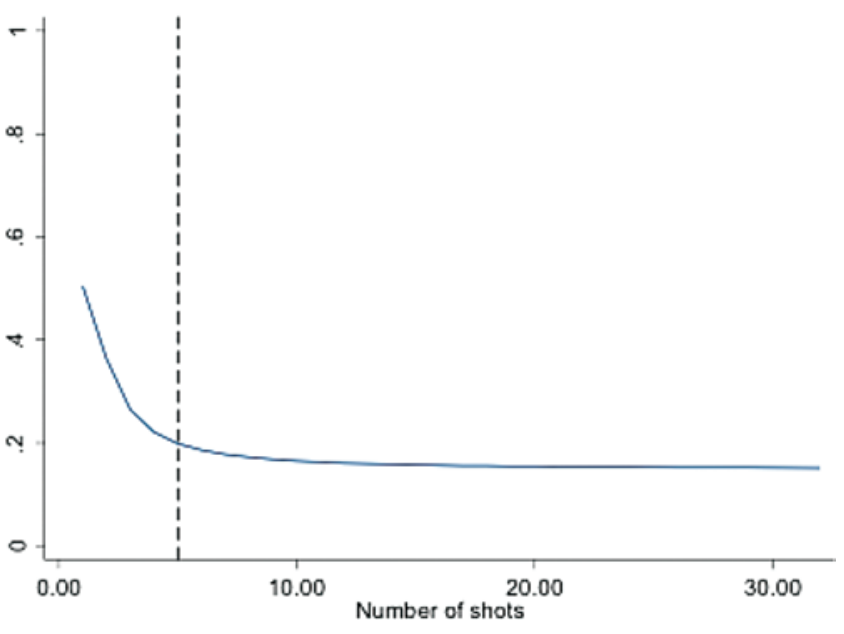

FIG. 4. Probability of presenting an acute effect depending on the number of shots during GKS.

\section{Radiation Dose Rate}

The radiation dose rate displayed a cutoff at $2.5 \mathrm{~Gy} /$ minute, with a decreased risk of developing an acute clinical ARE below this value, and a progressive increase for values more than $2.5 \mathrm{~Gy} /$ minute.

\section{Maximal Dose Received by the Cochlea and Vestibule}

In our series, based on previously published data ${ }^{15,19}$ for patients with functional hearing (GR Classes I and II), we tailored the dosimetry in order to keep the maximal dose to the cochlea below 5.2 Gy. Because of this, the gradient was modified toward other structures. In most of the cases, it involved the vestibule, which quite frequently received higher doses of radiation. If the impact of this on a long-term basis remains unknown, there is a tendency of presenting an acute effect in our series for a maximal dose of more than $8 \mathrm{~Gy}$. The relationship between the doses received by both structures - the cochlea and vestibule-is displayed in Fig. 8. Figure 9 additionally shows the different ranges of doses received by the vestibule: in 3 different instances, for a dose to the cochlea ranging between 4.2 and 5.2 Gy, the dose to the vestibule was 3.4 Gy, $6.9 \mathrm{~Gy}$, and $8.4 \mathrm{~Gy}$, respectively.

\section{Discussion}

To the best of our knowledge, all previous published studies have focused mainly on audiofacial and other $\mathrm{CN}$ morbidities during the medium- and long-term follow-up course after GKS. No study reported the acute effects and their management in patients presenting with acute and disabling symptoms during the first months after the treatment. As this problem may affect the patient's quality of life, we decided to investigate this issue.

The first case of VS was treated in 1969 by Leksellthe inventor of the Gamma Knife ${ }^{12}$-with a rather high dose of radiation (27 Gy at the margin). Due to the associated morbidities, mainly facial, there was a progressive de-
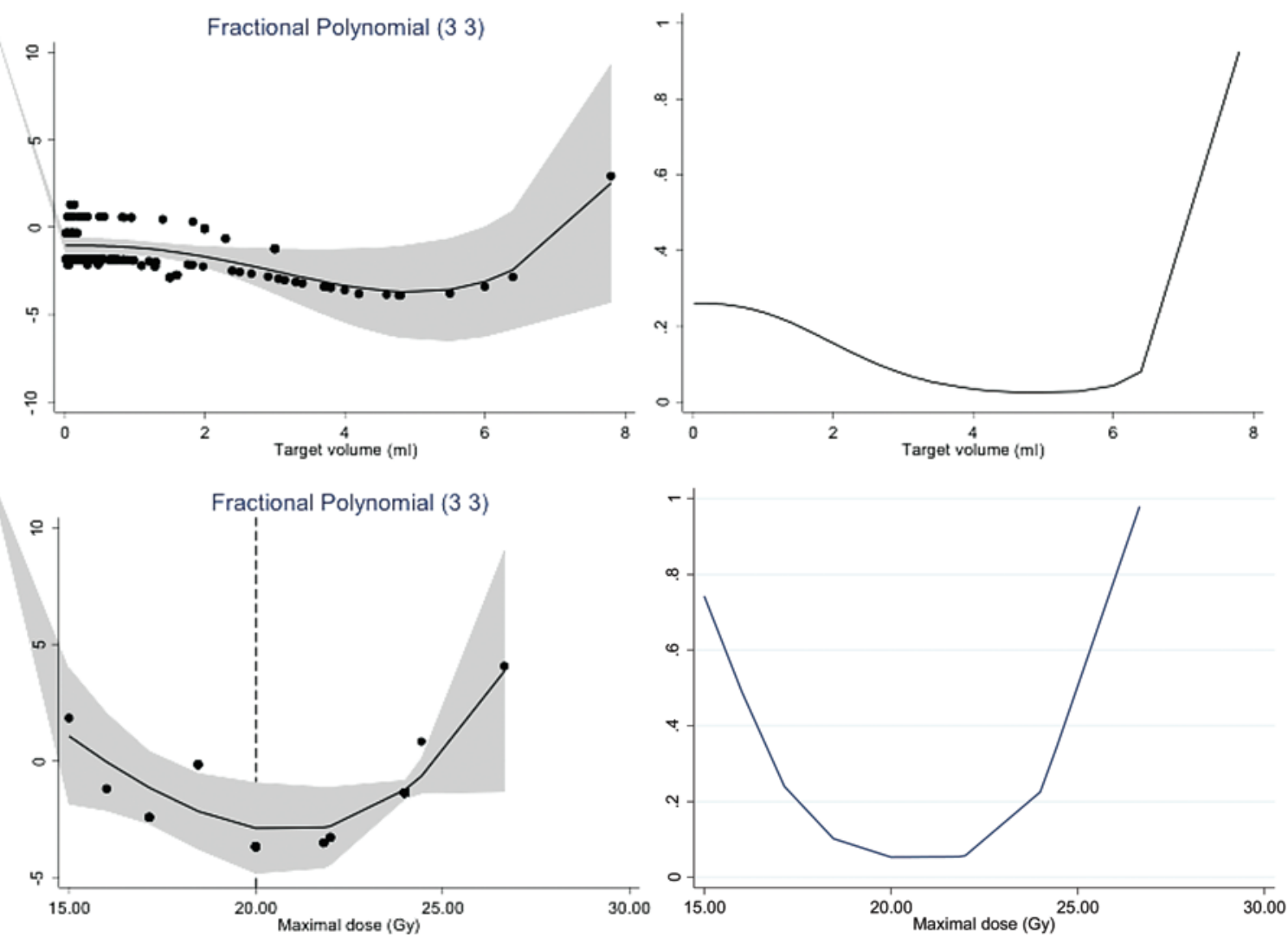

FIG. 5. Probability of presenting an acute effect depending on the TV and maximal dose prescribed during GKS. 

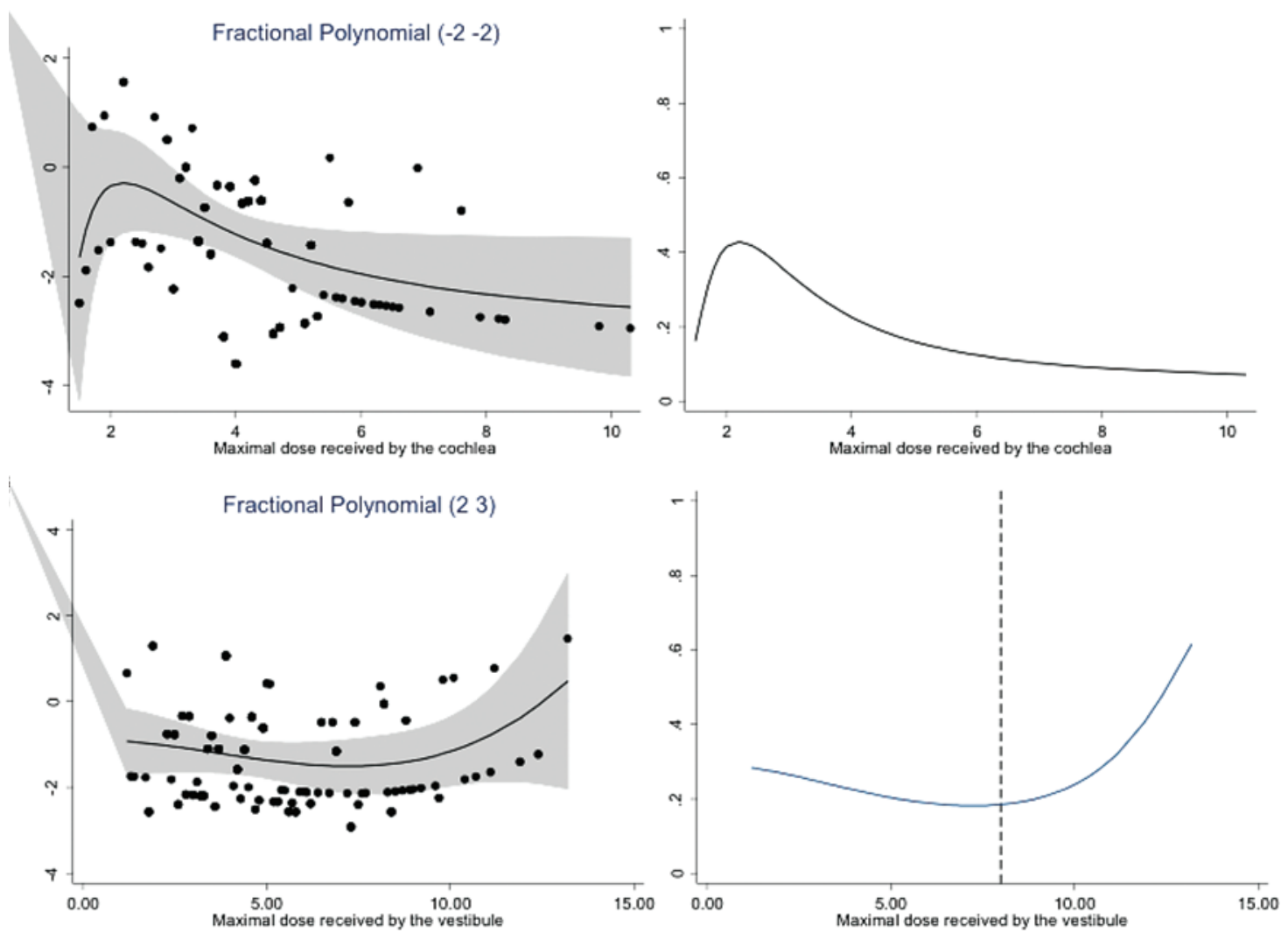

FIG. 6. Upper: Probability of presenting an acute effect depending on the maximal dose received by the cochlea. Lower: Probability of presenting an acute effect depending on the maximal dose received by the vestibule. The left panels show probability with confidence intervals, and the right panels display probability in a simplified manner, ranging from $0 \%$ to $100 \%$.

escalation of the doses, ${ }^{10}$ and the current typical prescription dose that is agreed upon by most of the teams is 12 to $13 \mathrm{~Gy}$ administered to the $50 \%$ isodose line. Technique refinement over time and the robotization of the Leksell Gamma Knife Perfexion also dramatically decreased the complications after GKS for VS. The primary aim of RS treatment in VS remains tumor control, which is attained by using GKS $(89 \%-98 \%)$ or the linear accelerator $(90 \%-$ 100\%). ${ }^{3,16}$ Additionally, the rates of facial nerve palsy became minimal-less than $1 \%$ with GKS in the Marseille experience-and virtually $0 \%$ since the robotization of GKS and the introduction of Leksell Gamma Knife Perfexion. ${ }^{17}$

It is rather difficult to evaluate all of the complications and their respective rates after GKS for VS due to the nonuniformity of measurements and follow-up periods and nonreporting of actuarial rates in lieu of crude ones. Usually, in the more modern series, authors focus on auditory morbidity. Vertigo and gait disturbance tend to have a more hazardous character and are difficult to quantify. A

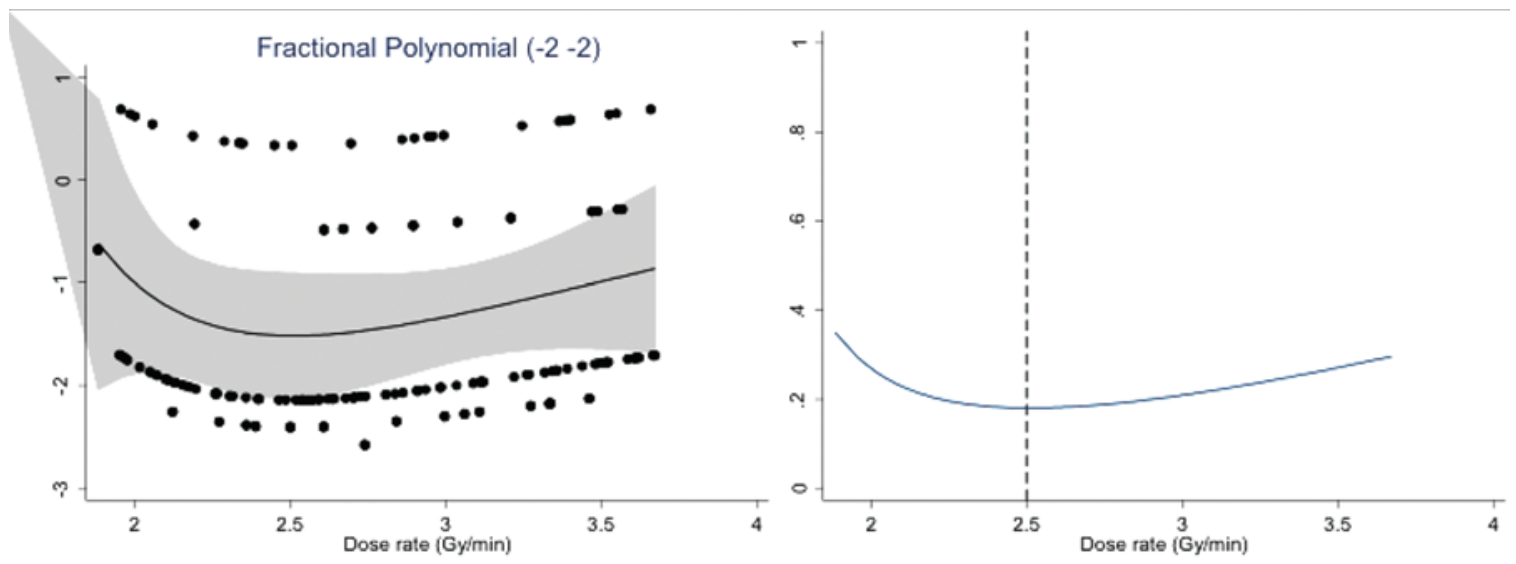

FIG. 7. Probability of presenting an acute effect depending on the radiation dose rate during GKS. Left: Probability is shown with confidence intervals. Right: Probability is shown in a simplified manner, ranging from $0 \%$ to $100 \%$. 


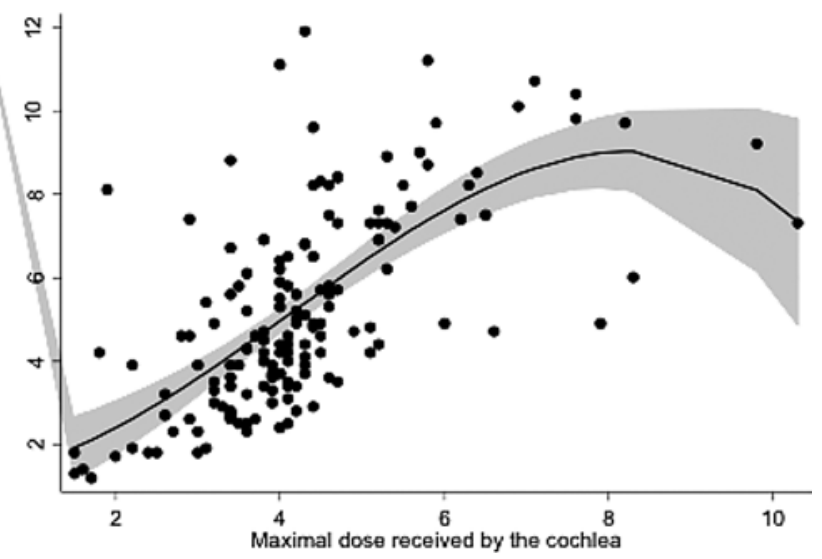

FIG. 8. The relationship between the dose received by the cochlea (continuous line) and the vestibule (dots) during GKS.

recent systematic review analyzed nonaudiofacial morbidity after GKS. ${ }^{20}$ The authors included 63 articles describing 5631 patients, of whom 3248 (58\%) patients received a mean radiation dose of less than 13 Gy and had a median follow-up of 39.5 months, and 2383 (42\%) patients received a mean radiation dose of more than 13 Gy and had a median follow-up of 36.5 months. Trigeminal neuropathy $(2.4 \%)$ - the most frequent complication-was found to occur more frequently in cases treated with more than 13 Gy (occurred over 28 times more). The occurrence of hydrocephalus, which is quite rare $(0.85 \%)$, was not influenced by the dose; however, symptomatic hydrocephalus that needed a shunt also displayed a cause-effect relationship at more than $13 \mathrm{~Gy}$. Vertigo or gait disturbance was reported in up to $1.1 \%$ of patients, with a higher prevalence in the lower dose cohort, which also applied to tinnitus (occurring in $0.1 \%$ ). In another recent study that provided data over a large cohort of 420 cases that were treated by GKS in the Netherlands, ${ }^{9}$ the authors reported that $1 \%$ of patients had new or increased permanent facial weakness, $3.1 \%$ of patients reported trigeminal neuropathy, $1.2 \%$ of patients had new or increased hydrocephalus, up to $13.2 \%$ of patients reported tinnitus, and $14.8 \%$ of patients reported new or increased vertigo. In the same study, serviceable hearing was preserved in $60.6 \%$ of cases.

In the present study, we focused on the appearance of acute clinical AREs that developed during the first 6 months after GKS and were reported by the patient as disabling symptoms. Of a total of 159 cases of VS treated with GKS (> 6 months of follow-up), 35 (22\%) patients had an acute clinical ARE. Among the de novo symptoms, the predominant ones were vertigo and gait disturbance, but, regarding the exacerbation of a preexisting symptom, this was more frequently related to auditory morbidity with the predominance of hearing loss. The clinical acute AREs disappeared in 32 (91.4\%) patients during the first 6 months after appearance. Usually, there was a marked improvement and/or disappearance during the week of corticosteroid treatment. In the univariate analysis, which was conducted with interest in the present study, the following variables were of interest: age; hearing at baseline (including PTA at baseline); Koos grade at baseline; number of the used isocenters; and the maximal dose received by the cochlea. Additional data assessed using fraction polynomial analysis suggest that for a developing clinical acute ARE, there might be an involvement of the maximal dose received by the tumor (cutoff at $20 \mathrm{~Gy}$, with an increased risk at more than $24 \mathrm{~Gy}$ ), number of shots (cutoff at 5 shots, with the same risk for $\geq 10$ shots), radiation dose rate (cutoff of $2.5 \mathrm{~Gy} /$ minute), and the vestibular dose (cutoff at 8 Gy). We do believe that the vestibular dose might play a major role, especially in developing vertigo and/or gait disturbances. Depending on the anatomical conditions, in order to protect the cochlea and administer a dose of no more than 5.2 Gy, we sometimes send the gradient toward the vestibule and thereby increase the dose received by the vestibule. Another potential hypothesis would involve the dose received by the ventral cochlear nucleus, as advocated by Linskey, ${ }^{14}$ which is more difficult to quantify and supposed to be less than or equal to $9 \mathrm{~Gy}$. However, regarding the ventral cochlear nucleus, in the present series most of the tumors were Koos Grade I and so probably the dose that was administered to the brainstem cochlear nucleus was minimal (actually close to zero). The limited sample size makes it difficult to achieve a clear statistical significance for some of the factors, as presented after the fractional polynomial analysis. A greater sample size would be necessary to show the clear and safe cutoff values needed to prevent any acute clinical ARE.

Additional quality-of-life questionnaires would have been of greater help to evaluate the outcomes of our patients. However, in the present setting, this has been difficult to organize.

\section{Conclusions}

Acute effects after RS for VS are not rare. In the present
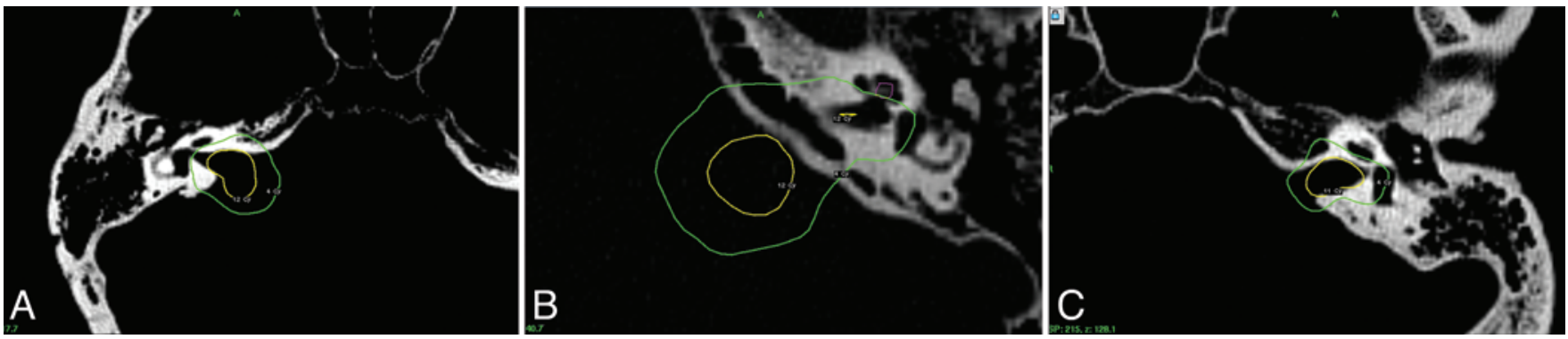

FIG. 9. CT scan images showing the dose received by the vestibule in different contexts of beam channel blocking, so as to keep the dose administered to the cochlea between 4.2 and 5.2 Gy. The doses received are $3.4 \mathrm{~Gy}(\mathbf{A}), 6.9 \mathrm{~Gy}(\mathrm{~B})$, and $8.4 \mathrm{~Gy}(\mathbf{C})$. 
study, we focused on their development during the first 6 months after GKS and reported by the patient as disabling symptoms. The dose prescribed at the margin in our series was 12 Gy. Thirty-five patients ( $22 \%$ of all patients treated by upfront GKS for VS during the same period of time) had an acute clinical ARE. In most of these cases, all of these acute effects were not permanent and ultimately improved or disappeared with the administration of steroids. Permanent clinical AREs remain very rare.

\section{Acknowledgments}

The authors acknowledge the help of Lausanne University Hospital.

\section{References}

1. Boari N, Bailo M, Gagliardi F, Franzin A, Gemma M, del Vecchio A, et al: Gamma Knife radiosurgery for vestibular schwannoma: clinical results at long-term follow-up in a series of 379 patients. J Neurosurg 121 Suppl:123-142, 2014

2. Chung WY, Liu KD, Shiau CY, Wu HM, Wang LW, Guo WY, et al: Gamma Knife surgery for vestibular schwannoma: 10-year experience of 195 cases. J Neurosurg 102 Suppl:87-96, 2005

3. Friedman WA, Bradshaw P, Myers A, Bova FJ: Linear accelerator radiosurgery for vestibular schwannomas. J Neurosurg 105:657-661, 2006

4. Gardner G, Robertson JH: Hearing preservation in unilateral acoustic neuroma surgery. Ann Otol Rhinol Laryngol 97:55-66, 1988

5. Hasegawa T, Kida Y, Kato T, Iizuka H, Kuramitsu S, Yamamoto T: Long-term safety and efficacy of stereotactic radiosurgery for vestibular schwannomas: evaluation of 440 patients more than 10 years after treatment with Gamma Knife surgery. J Neurosurg 118:557-565, 2013

6. Hayashi M, Chernov M, Tamura N, Izawa M, Muragaki Y, Iseki $\mathrm{H}$, et al: Gamma Knife robotic microradiosurgery for benign skull base meningiomas: tumor shrinkage may depend on the amount of radiation energy delivered per lesion volume (unit energy). Stereotact Funct Neurosurg 89:6-16, 2011

7. Hayashi M, Ochiai T, Nakaya K, Chernov M, Tamura N, Yomo S, et al: Image-guided microradiosurgery for skull base tumors: advantages of using gadolinium-enhanced constructive interference in steady-state imaging. J Neurosurg 105 Suppl:12-17, 2006

8. House JW, Brackmann DE: Facial nerve grading system. Otolaryngol Head Neck Surg 93:146-147, 1985

9. Klijn S, Verheul JB, Beute GN, Leenstra S, Mulder JJ, Kunst HP, et al: Gamma Knife radiosurgery for vestibular schwannomas: evaluation of tumor control and its predictors in a large patient cohort in The Netherlands. J Neurosurg 124:1619-1626, 2016

10. Kondziolka D, Lunsford LD, McLaughlin MR, Flickinger JC: Long-term outcomes after radiosurgery for acoustic neuromas. N Engl J Med 339:1426-1433, 1998

11. Kondziolka D, Mousavi SH, Kano H, Flickinger JC, Lunsford LD: The newly diagnosed vestibular schwannoma: radiosurgery, resection, or observation? Neurosurg Focus 33(3):E8, 2012

12. Leksell L: A note on the treatment of acoustic tumours. Acta Chir Scand 137:763-765, 1971

13. Lin D, Hegarty JL, Fischbein NJ, Jackler RK: The prevalence of "incidental" acoustic neuroma. Arch Otolaryngol Head Neck Surg 131:241-244, 2005
14. Linskey ME: Hearing preservation in vestibular schwannoma stereotactic radiosurgery: what really matters? J Neurosurg 109 Suppl:129-136, 2008

15. Massager N, Nissim O, Delbrouck C, Delpierre I, Devriendt D, Desmedt F, et al: Irradiation of cochlear structures during vestibular schwannoma radiosurgery and associated hearing outcome. J Neurosurg 107:733-739, 2007

16. Meijer OW, Weijmans EJ, Knol DL, Slotman BJ, Barkhof F, Vandertop WP, et al: Tumor-volume changes after radiosurgery for vestibular schwannoma: implications for follow-up MR imaging protocol. AJNR Am J Neuroradiol 29:906910, 2008

17. Régis J, Carron R, Delsanti C, Porcheron D, Thomassin JM, Murracciole X, et al: Radiosurgery for vestibular schwannomas. Neurosurg Clin N Am 24:521-530, 2013

18. Régis J, Carron R, Park MC, Soumare O, Delsanti C, Thomassin JM, et al: Wait-and-see strategy compared with proactive Gamma Knife surgery in patients with intracanalicular vestibular schwannomas. J Neurosurg 113 Suppl:105-111, 2010

19. Régis J, Tamura M, Delsanti C, Roche PH, Pellet W, Thomassin JM: Hearing preservation in patients with unilateral vestibular schwannoma after gamma knife surgery. Prog Neurol Surg 21:142-151, 2008

20. Sughrue ME, Yang I, Han SJ, Aranda D, Kane AJ, Amoils $M$, et al: Non-audiofacial morbidity after Gamma Knife surgery for vestibular schwannoma. Neurosurg Focus 27(6):E4, 2009

21. van de Langenberg R, Hanssens PE, van Overbeeke JJ, Verheul JB, Nelemans PJ, de Bondt BJ, et al: Management of large vestibular schwannoma. Part I. Planned subtotal resection followed by Gamma Knife surgery: radiological and clinical aspects. J Neurosurg 115:875-884, 2011

\section{Disclosure}

The authors report no conflict of interest concerning the materials or methods used in this study or the findings specified in this paper.

\section{Author Contributions}

Conception and design: Tuleasca, Levivier. Acquisition of data: Tuleasca, George, Schiappacasse, Zeverino, Maire, Levivier. Analysis and interpretation of data: Tuleasca, George, Faouzi, Schiappacasse, Zeverino, Maire, Levivier. Drafting the article: Tuleasca, Faouzi, Levivier. Critically revising the article: all authors. Reviewed submitted version of manuscript: all authors. Approved the final version of the manuscript on behalf of all authors: Tuleasca. Statistical analysis: Faouzi. Administrative/ technical/material support: Tuleasca, Faouzi, Schiappacasse, Maire, Levivier. Study supervision: Levivier.

\section{Supplemental Information Previous Presentations}

Portions of this paper were presented in an oral presentation form at the 18th Leksell Gamma Knife Society Meeting in Amsterdam, The Netherlands, on May 18, 2016.

\section{Correspondence}

Constantin Tuleasca, Centre Hospitalier Universitaire Vaudois, Neurosurgery Service and Gamma Knife Center, Rue du Bugnon 44-46, BH-08, Lausanne CH-1011, Switzerland. email: constantin.tuleasca@gmail.com. 